\title{
LA REPRESENTACiÓN DE LA MujER Y LOS IDEALES DEL PENSAMIENTO Colombiano de Finales del SiglO XIX y COMIENZOS DEL SigLO XX: LA Metáfora de la Falsa InClusión**
}

Fecha de recepción: 22 de agosto de 2013 Fecha de aprobación: 2 de diciembre de 2013

\section{Resumen}

A finales del siglo XIX y comienzos del siglo XX el proceso de construcción de la nación colombiana transitó por dos caminos adversos: el Periodo Radical -de corte liberal- y el Periodo de la Regeneración -de tinte conservador-. A partir de este contexto histórico-social se analiza la representación que se hace de la mujer durante este cambio de siglo, con el ánimo de evidenciar que el imaginario de género que se construyó allí está marcado por la metáfora de la falsa inclusión, es decir, que cada periodo a través de la noción que configura del poder, incluye a la mujer para, paradójicamente, excluirla. A partir de dicha metáfora este texto termina con un breve acercamiento a la propuesta de Tomás Carrasquilla y su personaje de Bárbara Caballero en La Marquesa de Yolombó y cómo este muestra la diatriba a la que se enfrentaba la mujer: tradición vs. independencia.

Palabras clave: representación de la mujer, metáfora, falsa inclusión.

* Artículo de reflexión, avance de la Investigación: "La Mujer en la Literatura colombiana".

Citar: Suárez Reina, A. (Enero - Junio de 2014). La Representación de la Mujer y los Ideales del Pensamiento Colombiano de Finales del Siglo XIX y Comienzos del Siglo XX: la Metáfora de la Falsa Inclusión. La Palabra (24), 33 - 41

\section{Adriana Yamile Suárez Reina}

Universidad Libre, Colombia Universidad Distrital Francisco José de Caldas, Colombia aysuarezr@gmail.com

Licenciada en Lingüística y Literatura de la Universidad Distrital Francisco José de Caldas. Magíster en Lingüística Española del Instituto Caro y Cuervo. Docente de la Universidad Libre y la Universidad Distrital Francisco José de Caldas. Bogotá, Colombia. 


\title{
The Representation of Woman and the Ideals of Colombian Thought IN THE END OF THE XIX Century: the Metaphor of False Inclusion
}

\begin{abstract}
At the end of XIX century and the beginning of XX century the nation building process passed through two adverse paths: the radical period (a liberal perspective), and the Regeneration period (a mostly conservative one). Based on this historical and social context, this paper analyzes the representation of woman during this turn of century, and aims at showing that the gender imaginary constructed in this period was primarily influenced by the metaphor of false inclusion. In each of these historical periods: we find a configured notion of power that includes woman in order to, paradoxically, exclude her. Based on the above mentioned metaphor, this paper ends with a short approach to Tomás Carrasquilla's position and the character Barbara Caballero in La marquesa de Yolombó, showing how this author presents the diatribe woman was confronted with: tradition versus independence.
\end{abstract}

Key words: representation - women, metaphor, false inclusion.

\section{LA RepréSENTATION de LA FEMME ET DES IdÉAUX de la Pensèe Colombienne de la Fin du Xixème SiÈcle et Début du XXème Siècle: la Metaphore DE LA FAUSSE INCLUSION}

\section{Résumé}

À la fin du XIXème siècle et au début du XXème siècle, le processus de construction de la nation colombienne a transité par deux chemins adverses : la période radicale -côté libéral- et la période de la régénération - principalement conservateur-. À partir de ce contexte historico-social, ce travail analyse la représentation que l'on fait de la femme, pendant ce changement de siècle, avec l'intention de mettre en évidence que l'imaginaire de genre que l'on y construit, est principalement marqué par la métaphore de la fausse inclusion ; c'est-à-dire que chaque période, à travers la notion qui configure du pouvoir, inclut la femme pour, paradoxalement l'exclure. À partir de cette métaphore-là, ce texte termine avec un bref approchement de la proposition de Tomás Carrasquilla et son personnage de Bárbara Caballero dans La Marquise de Yolombo et comment celui-ci montre la diatribe à laquelle doit faire face la femme : tradition vs indépendance.

Mots Clés: représentation de la femme, métaphore, fausse inclusion. 


\section{Introducción}

Abordar las problemáticas en torno al proyecto de construcción de nación en Colombia ha significado un gran desafío para todas las disciplinas que han intentado dar cuenta de dicho proceso, más aún si dentro de este abordaje se busca vincular los ideales del pensamiento colombiano de finales del siglo XIX y comienzos del siglo $\mathrm{XX}$ con la representación de la mujer que allí se gestó. Este artículo se centra en la reconstrucción de la imagen femenina de la época a través del análisis de las condiciones que dieron pie a dicha representación; cabe aclarar que esta no se concibe solamente desde una mirada biológica referida al sexo, sino que se retoma desde la noción de género, entendida con McConnell-Ginet (1992) como el conjunto de fenómenos sociales, culturales y psicológicos que se encuentran asociados a este. Así, esta mirada se dirige a la representación de la mujer no como condicionante del discurso ideológico de la época sino como su producto.

El análisis de la representación de la mujer en esta época remite entonces a los sistemas de valores, ideas y prácticas que permitín, por un lado, establecer un orden para aprehender el mundo social y, por otro, posibilitar la comunicación entre los miembros de una comunidad por medio de un código que regulara unitariamente los diversos aspectos de su entorno social. De acuerdo con lo anterior, el presente texto abordará, en primera instancia, un contexto histórico-social en lo referido a las ideologías imperantes en la Colombia de finales del siglo XIX y comienzos del siglo $\mathrm{XX}, \mathrm{y}$, en un segundo momento, establecerá las relaciones entre dichas ideologías y el resultado de la representación de la mujer, para finalmente establecer algunas de las líneas de análisis que subyacen a lo planteado por Carrasquilla en La Marquesa de Yolombó.

\section{La diatriba liberal- conservador}

En el tránsito entre el siglo XIX y el XX, el proyecto de nación en Colombia atravesó por dos caminos disímiles: el Radicalismo Liberal y la Regeneración. El primero de ellos, concebido como una corriente política que se mostró partidaria de reformas extremas al declararse en contra de las dictaduras y el autoritarismo; el segundo, por su parte y como su nombre lo indica, buscó la reconstrucción del país a través de un llamado a la unidad nacional.

Es hacia mediados del siglo XIX donde se originó el Radicalismo Liberal amparado en la Constitución de 1853. Allí se inauguró el periodo del federalismo caracterizado por la libertad administrativa de los Estados constituyentes y por la separación de la Iglesia de los entes gubernamentales. Sin embargo, fue hasta la Constitución de 1863, denominada Constitución de Rionegro, cuando este movimiento tomó mayor fuerza; en esta carta magna se establecieron las libertades fundamentales inspiradas en la Revolución Francesa, se hizo hincapié en la secularización del Estado, se propendió por la educación oficial, por la formación de docentes y se cambió el nombre del país de Confederación Granadina a Estados Unidos de Colombia, ratificando con ello el sistema federal de gobierno.

Este Olimpo Radical, -en el cual se incluyen personajes como Manuel Murillo Toro, Aquileo Parra, Tomás Cipriano de Mosquera, por solo mencionar algunos nombres-, contrario a las tradiciones, defendió el liberalismo. Su objetivo: llevar a Colombia a la modernización, hacer partícipe al país de los logros de la Revolución Industrial y sus adelantos tecnológicos. En esa medida, se consideraba que la salida a los problemas del país estaba en la industrialización, de ahí que en este periodo se diera paso a la construcción de ferrocarriles, empresas de telégrafos, bancos, para tratar de alcanzar las ventajas de dichos cambios. Asimismo, en este periodo se generó una preocupación por la educación laica, ya que se 
crearon varias instituciones de este carácter, tales como las Normales, el establecimiento mediante ley de la Universidad Nacional de Colombia y la Academia Colombiana de la Lengua; sin embargo, este sueño del "Olimpo" despertó grandes contradictores.

Hacia 1875, y aprovechando la crisis del Radicalismo Liberal causada por el manejo de las exportaciones agrícolas, los opositores conservadores orientados por Miguel Antonio Caro se fueron fortaleciendo gracias a la alianza de los disidentes radicales en torno a la figura de Rafael Núñez. Un año después el enfado de los conservadores y el clero por las reformas a la educación hizo estallar la guerra civil en Cauca, Antioquia y Tolima, situación que catapultó el movimiento de la Regeneración, cuyo objetivo fundamental era eliminar el desorden político y administrativo que supuestamente había creado el régimen federal. Al respecto, Palacios y Safford afirman:

El lapso de 1878 a 1900 se conoce como la Regeneración. El nombre deriva de una frase de Rafael Núñez (...) En 1878, Núñez resumió la crítica al periodo radical diciendo que había puesto a los colombianos ante la alternativa de "regeneración administrativa fundamental o catástrofe". En los años siguientes de- sarrolló el planteamiento de este modo: el federalismo y el doctrinalismo radical extremos habían llevado al país a la "catástrofe" de la que saldría mediante el advenimiento del "fecundo reinado de la paz científica". Colombia necesitaba una Constitución centralista que reconociera en principio el catolicismo como elemento medular de cohesión social (2012, p. 350).

Con estas palabras de Núñez se inicia entonces el proyecto de regenerar al país, programa que alcanza su auge en la redacción de una nueva carta magna para Colombia: la Constitución de 1886. Desde allí se centraliza la política, el Estado interviene en la economía, el país se transforma en República y de nuevo el catolicismo toma su papel de co-gobierno, pues se le encomienda proteger la educación en el territorio nacional.

La Regeneración no fue solamente un llamado a reconstruir el país, fue una metáfora que marcó el discurso político de la época y, por ende, las acciones de sus ciudadanos. Este proyecto de consolidar el Estado-Nación a través de una perspectiva hegemónica fue una mirada reflexiva de dos aspectos que se encuentran mal: la política y la población. Regenerar, entonces, en tanto metáfora hace pensar en la nostalgia por un pasado perdido al que hay que regresar y que es preciso renovar, todo ello "en nombre de Dios, fuente suprema de toda autoridad" (Melgarejo, 2008, p. 280).

\section{La representación} de la mujer: su falsa inclusión

Los proyectos de nación abordados anteriormente, a pesar de mostrar elementos disímiles en sus aspectos ideológicos (liberalismo vs. conservadurismo), comparten, respecto a la visión de la mujer, la metáfora de la falsa inclusión. Cada programa, a través de la noción que configura del poder, la incluye para excluirla: en el Radicalismo Liberal si bien se dio paso a contemplar la problemática de la desigualdad -lo cual creó un ambiente de mayor libertad- escasamente se problematizó la sujeción de un género sobre otro, pues con ese sofisma de distracción se invisibilizó el problema (Bermúdez, 1993). En la Regeneración este entorno de libertad se limitó al ser su premisa la necesidad de homogeneizar a través de la disciplina o el encauzamiento de quienes iban por "mal camino"; la mujer se concibe entonces como ese "ser otro" fuera de la norma, hecho que justificó su subordinación. Para ejemplificar esta metáfora se hará un recorrido por tres aspectos: los discursos desautorizadores, la vulnerabilidad y el aislamiento y, por último, la normalización ${ }^{1}$.

1 Estas categorías se retoman de los trabajos que analizan la exclusión social de la mujer en el ámbito de la discapacidad (Universidad de Barcelona, s.f.); no obstante, en este texto se presentan desde una adaptación propia. 
El Radicalismo Liberal creó un discurso que avalaba el desarrollo de las libertades fundamentales para la mujer. En este marco, dicho discurso daba un mayor campo de acción al género femenino pero si se salía de este, se desautorizaba y se cuestionaba su rol. Por ejemplo, se buscó limitar el poder de influencia de la Iglesia sobre la mujer, razón por la cual se propendió por una educación laica; sin embargo, el objetivo de esta educación no era que las mujeres conocieran el humanismo, los avances de la ciencia ni mucho menos que desarrollaran su pensamiento crítico. Por el contrario, lo que se buscaba era capacitar a las mujeres para que desempeñaran "más profesionalmente" su rol en el hogar; así, "los oficios aceptados eran ser maestras, costureras, voluntarias, al igual que las labores manuales y la pintura. Si en algún caso la mujer decidía trabajar fuera de la casa durante su tiempo libre debía tratar de no perder su feminidad" (Bermúdez, 1987, p. 76). De esta manera, la libertad de la mujer no se daba en términos de la elección sino de su participación como pieza clave del proyecto de nación.

La mujer entonces se capacitó en la economía doméstica, leyó textos propios para su sexo y participó en política al replicar las ideas de padres, hermanos o esposos. Cabe anotar que todo esto se hizo sin salir por lapsos pro- longados del hogar ya que no siempre la educación se dio en centros educativos. El objetivo del Radicalismo Liberal era optimizar el uso del tiempo del "bello sexo" al desplazarlo de la Iglesia al hogar sin que se cambiara por el tiempo de la iglesia a la escuela. Precisamente, la mujer se concibió con una función: crear buenos ciudadanos aunque a ella misma no se le confiriera este estatus.

En la misma línea, la Regeneración también evidenció un discurso desautorizador en tanto que, aunque buscó la integración cultural y la cohesión social, creó políticas que tenían una injerencia directa en la vida de los pobladores:

La necesidad de "limpiar" la lengua a través de la gramática, el alma a través de la moral y el cuerpo a través de los manuales de urbanidad hace parte de todo un programa de regeneración nacional que se pondría en vigencia en varios contextos nacionales. A través de esta idea se construye un discurso político con una comprensión particular de la vida social ligada a los centros de poder estatal, y unas políticas sobre el cuerpo y el comportamiento correspondientes a la formación de sujetos nacionales, así como modos específicos de reconocer la población y el territorio (Melgarejo, 2008, p. 281).

La mujer entonces se integró socialmente no desde su individualidad sino desde su "re-educación" a través de la religión y los manuales que se le imponían. Esta educación confesional hizo que las mujeres comulgaran con valores como el del prediseño de una vida buena que debían perseguir, es decir, un deber ser caracterizado altamente por ser buenas representantes de su sexo -femineidad-, el ser buena ama de casa, ser buena esposa y, por su puesto, ser una mujer casta y devota. Así pues, los discursos -liberales y conservadores- aunque tienen en cuenta a la mujer, circunscriben su actuación para que esta sea benéfica para el proyecto de nación. El discurso las autoriza a ejercer su libertad, pero desautoriza en sí mismo su libertad de acción.

De otro lado, la vulnerabilidad y el aislamiento son elementos que ejemplifican el uso de la metáfora de la falsa inclusión de la mujer en los proyectos de nación en Colombia. Es necesario aclarar que la vulnerabilidad se entiende no solo como la posibilidad de ser herido ya sea física o moralmente, como lo menciona la RAE, sino desde un sentido negativo que alude a las limitaciones asociadas a la condición de mujer, tales como la falta de autonomía (no ser capaz de valerse por sí misma) y la necesidad de una protección especial durante toda la vida, lo cual conlleva a un aislamiento de la mujer en la vida social. 
Respecto al Radicalismo, si bien la mujer posee un poco más de reconocimiento, sigue siendo una figura vulnerable $y$, por ende, debe aislarse o resguardarse en el calor del hogar. Tal y como lo señala Bermúdez quien afirma que durante el Olimpo Radical la mujer aprendió con la educación a desarrollar mejor su trabajo como ama de casa, pero no podía desarrollar nada fuera de este ámbito; su inteligencia era reconocida, pero por su condición de mujer podía ser presa del peligro que esta representaba y perder su esencia femenina:

- Debía ser justa en la casa, pero sin dejar de guardar las distancias establecidas con sus dependientes (sirvientes e hijos), y así mismo debía aceptar pasivamente su posición subordinada en la sociedad.

- Debía efectuar el trabajo doméstico con gran orden y eficacia, pero estaba incapacitada para desarrollar otras labores fuera del hogar que no se relacionaran con el mundo femenino.

- El uso del tiempo libre, ya no sólo se debía limitar a las oraciones, sino que se les exigía estudiar y trabajar para lograr mujeres más independientes, pero sin olvidar sus obligaciones femeninas.

- Los problemas de tristeza, tedio, melancolía y/o cansancio que vivían algunas damas en el hogar se podían solucionar con resignación, orden y disciplina en la casa, evitando el ocio y llevando un régimen higiénico y sano: pero no se debían averiguar o discutir las razones de tales preocupaciones (1987, p. 82).

En la lista anterior se aprecia la ambivalencia del proyecto liberal en el cual la mujer, aunque ganaba algunos beneficios, ganaba asimismo algunas restricciones. En ese orden de ideas, es paradójico que este proyecto de nación estimulado por los ideales de la Revolución Francesa -libertad, igualdad y fraternidad- no haya inquirido por las relaciones entre géneros y nunca haya cuestionado el sometimiento de la mujer al hombre. De esta manera, es posible decir que el Olimpo Radical construyó su propia versión interpretativa del liberalismo, puesto que la mujer era vista desde su vulnerabilidad o propensión a padecer conflictos y la respuesta del proyecto de nación no podía ser otra que generar un espacio acorde con sus limitadas posibilidades de acción.

Para el caso del discurso de la Regeneración, las nociones de vulnerabilidad y aislamiento no se dieron de manera encubierta como en el periodo del Olimpo Radical; por el contra- rio, en esta época se reconocía abiertamente un papel inferior de la mujer frente al hombre, todo ello amparado en la encíclica Syllabus Errorum ${ }^{2}$ de Pío IX. En este paradigma conservador, la Iglesia recobró su poder $y$, en consecuencia, esta presentación del Estado como confesional configuró la visión de la mujer desde su fragilidad. En esa línea, el carácter religioso fue un imperativo en la vida de la mujer por su tendencia bíblica al pecado ${ }^{3}$.

La concepción de la mujer decayó. La subordinación estaba plenamente justificada y, además, amparada en la Ley; por ejemplo, en el Código Civil colombiano expedido por el Congreso de 1873 y adoptado por la Ley 57 de 1887, se da cuenta de esta situación en los artículos: 195, si la casada ejerce industria se supone autorización del marido para los actos consiguientes; 2347, de sus hechos, siendo casada, es en ciertos casos responsable el marido; 2502 a 2504, la casada es acreedora privilegiada de cuarta clase por los bienes de su propiedad que administra el marido, entre otros. En comparación con el periodo anterior, en el cual el matrimonio desde la perspectiva liberal, se concebía como un contrato social, en este nuevo régimen la mujer

2 Este documento publicado en 1864 llevaba como subtítulo "Catálogo que comprende los principales errores de nuestra época señalados en las encíclicas y otras caras apostólicas de nuestro santísimo señor Pío Papa IX".

3 Recordemos el pasaje bíblico alusivo al pecado original en el que Dios le dice a Eva: "Multiplicaré tus sufrimientos en los embarazos. Con dolor darás a luz a tus hijos, necesitarás de tu marido y él te dominará” (La Biblia, Catholic.net. Génesis, Capítulo 3 , versículo 16). 
se transformó en un ser incapaz jurídicamente, similar a los niños o a los dementes (Velásquez, 1995, p. 183).

En esa misma línea se da la normalización, entendida como el proceso de regulación para que la mujer desempeñe su rol en el grupo social. Como ya se ha mencionado, en ambos periodos se genera esta regulación ya sea desde la educación o desde la legislación gubernamental; solo para recordar, en primera instancia el Radicalismo hace uso de los códigos civiles redactados según cada Estado federal en donde uno de los elementos más importantes fue el del divorcio, pues el carácter laico favorecía esta posición. Del mismo modo, en lo referido a la educación, la mujer moderna garantizó el vínculo entre la familia y el Estado, ordenó la sexualidad en el hogar, afinó su comportamiento individual al racionalizar el gasto y aprovechar los recursos de la hacienda, es decir, la mujer hacendosa estaba capacitada para gestionar tanto la riqueza del hogar como del país (Pedraza, 2011). En cuanto a la Regeneración, se da prelación a la educación a través de lecturas formativas que aconsejan la actuación como hija, esposa y madre: Consejos de un padre a su bija, Educación de la mujer, La coqueta, La hermosura, Reflexiones para las señoritas, Un matrimonio en proyecto, entre otros.

\section{Tomás Carrasquilla y La Marquesa de Yolombó}

Tomás Carrasquilla (18581940) es un escritor antioqueño que ha sido denominado como uno de los más importantes en la literatura colombiana. En este caso no se busca discutir la pertenencia de este autor a una u otra categoría, simplemente se retoma, de forma sucinta, $L a$ Marquesa de Yolombó, no desde el tiempo interno de la obra -descrito en la novela desde un ambiente colonial- sino desde su momento de enunciación (1926), el cual está conectado con la diatriba liberal-conservadora que se expuso líneas más arriba; esto con el ánimo de reflexionar a propósito de la representación de la mujer desde el personaje de Bárbara Caballero.

Carrasquilla, a través del personaje de la Marquesa, señala los alcances de esta mujer independiente y entre tanto hace énfasis en el conflicto que estos le generan. En esa línea se hace evidente la metáfora de la falsa inclusión, puesto que aparecen los discursos desautorizadores; por un lado se proclama la educación, pero con la restricción de la formación para el hogar: "unas miajas de doctrina, las oraciones del diario, dos o tres para las grandes solemnidades, enseñadas y aprendidas por el método consabido e inmortal del loro; el consejo de que fuesen muy buenas y muy señoras de su casa y de que cumpliesen «los deberes de obligación del estado»" (Carrasquilla, 2008, p. 114). Asimismo, se hace patente la vulnerabilidad y el aislamiento respecto a la debilidad de la mujer y el manejo de su inteligencia: "No ve, Chata: si saben leer, pueden aprender cosas muy malas, en los libros; si saben escribir, pueden cartearse con hombres malos, que están por perjudicarlas" (p. 45).

A pesar de que la Marquesa aprende a leer esto no le impide que la aíslen de su ser mujer: "Ya ves que piensa con el juicio y la cordura del hombre más capaz" (Carrasquilla, 2008, p. 53). Finalmente, la normalización se da cuando Doña Bárbara abraza de nuevo la religiosidad: "a la Marquesa la ha sepultado Dios en la locura, para resucitarla a la santidad" (p. 459). Así las cosas, doña Bárbara Caballero fue una mujer excepcional para su época; aprovechó lo que le proporcionó la educación y por esto pudo llevar con éxito el negocio de la minería; además, a pesar del influjo que poseía en ella la razón, era supersticiosa y, al igual que todas las mujeres, no es inmune a los desencantos del amor, lo que la llevó a caer en la locura para reconocer las limitaciones de su libertad. 
La posición de Carrasquilla no era reivindicar a la mujer, por el contrario pretendía mostrar un personaje acorde con la época, por tanto, Doña Bárbara Caballero encarna la metáfora de falsa inclusión, pues a pesar de que el idealismo es lo que la caracterizaba, las libertades que creía poseer fueron las directas responsables de su crisis, la cual resolvió a través de una verdadera exclusión.

\section{Conclusiones}

La representación de la mujer y los ideales del pensamiento colombiano de finales del siglo XIX y comienzos del siglo XX, tal y como se presentó a lo largo del texto, se encuentran íntimamente ligados, en tanto que dicha representación es producto de los proyectos de nación de las épocas estudiadas al compartir la metáfora política de la falsa inclusión. A través de esta metáfora se da cuenta del papel de la mujer y la poca preocupación por analizar las relaciones de poder con otros géneros, así como el énfasis que se da en las funciones que se le adjudicaron respecto a la formación del Estado.

Si bien el Olimpo Radical le dio a la mujer un contexto de mayor libertad, su campo de acción pocas veces traspasó el hogar y sus labores asociadas. Por su parte, el proyecto de la Regeneración -desde sus deseos centralistas y reunificadores- dio un retroceso en la representación de la mujer, dado que esta nuevamente se instituyó como el centro del hogar. Esta metáfora de la falsa inclusión como representación de la mujer es a lo que se enfrentó el personaje de Doña Bárbara Caballero y Alzate, pues se alimentó del conocimiento que proclamaba el liberalismo y sufrió con el status quo que reclamaba con vehemencia el esencialismo del "bello sexo".

\section{Referencias}

Bermúdez, S. (1987). Mujer y familia durante el Olimpo Radical”. Anuario Colombiano de la Historia Socialy de la Cultura, 15, 57-90. Bogotá.

Bermúdez, S. (junio-diciembre, 1993). El "bello sexo" y la familia durante el siglo XIX en Colombia. Revisión de publicaciones sobre el tema. Historia Crítica, 8, 34-51.

Carrasquilla, T. (2008). La Marquesa de Yolombó. Bogotá: Alfaguara.

McConnell-Ginet, S. (1992). "Lenguaje y género". En F. Newmeyer (Ed.), Panorama de la Lingüística Moderna de la Universidad de Cambridge IV. El lenguaje: contexto socio-cultural (pp. 99 - 126). Cambridge: Cambridge University Press.

Melgarejo, M. (2008). “Trazando las huellas del lenguaje político de La Regeneración: la nación colombiana y el problema de su heterogeneidad excepcional”. En S. Castro y E. Restrepo (Ed.), Genealogías de la colombianidad. Formaciones discursivas y tecnologias de gobierno en los siglos XIX y XX. Bogotá: Pontificia Universidad Javeriana, Instituto de Estudios Sociales y Culturales Pensar.

Ministerio de Trabajo y Asuntos Sociales y Universidad Autónoma de Barcelona (s.f.). Indicadores de exclusión social de mujery discapacidad. Recuperado de http://www.capaces.org/upload/fckeditor/File/indicadores_exclusion_mujer_discap.pdf 
Palacios, M. y Safford, F. (2012). Historia de Colombia. País fragmentado, sociedad dividida. Bogotá: Universidad de los Andes.

Pedraza, Z. (diciembre, 2011). "La educación de las mujeres": el avance de las formas modernas de la feminidad en Colombia. Revista de Estudios Sociales, 41, 72-83.

Código civil colombiano expedido por el Congreso de 1873 y adoptado por la Ley 57 de 1887: con un suplemento de las leyes que lo adicionan y reforman, desde 1887 hasta 1892, inclusive (1895). Bogotá: Imprenta Nacional.

Velásquez, M. (1995). "La república liberal y la lucha por los derechos civiles y políticos de las mujeres". En Consejería Presidencial para la Política Social y Presidencia de la República de Colombia, Las mujeres en la bistoria de Colombia. Tomo I: mujeres, historia y política. Santafé de Bogotá: Editorial Norma. 